\title{
Complex Valued $b$-Metric Spaces and Common Fixed Point Theorems under Rational Contractions
}

\author{
Anil Kumar Dubey \\ Department of Mathematics, Bhilai Institute of Technology, Bhilai House, Durg, Chhattisgarh 491001, India \\ Correspondence should be addressed to Anil Kumar Dubey; anilkumardby@rediffmail.com
}

Received 16 November 2015; Revised 2 March 2016; Accepted 10 May 2016

Academic Editor: Arcadii Z. Grinshpan

Copyright (C) 2016 Anil Kumar Dubey. This is an open access article distributed under the Creative Commons Attribution License, which permits unrestricted use, distribution, and reproduction in any medium, provided the original work is properly cited.

The aim of this paper is to prove the existence and uniqueness of a common fixed point for a pair of mappings satisfying certain rational contraction conditions in complex valued $b$-metric space. The obtained results generalize and extend some of the wellknown results in the literature.

\section{Introduction}

Banach contraction principle in [1] gives appropriate and simple conditions to establish the existence and uniqueness of a solution of an operator equation $T x=x$. Later, a number of papers were devoted to the improvement and generalization of that result. Most of these results deal with the generalizations of the different contractive conditions in metric spaces. There have been a number of generalizations of metric spaces such as vector valued metric spaces, $G$ metric spaces, pseudometric spaces, fuzzy metric spaces, $D$-metric spaces, cone metric spaces, and modular metric spaces. Bakhtin [2] introduced the notion of $b$-metric space which is a generalized form of metric spaces.

In [3], Czerwik proved the contraction mapping principle in $b$-metric spaces. Subsequently, many authors obtained fixed point results for single valued and multivalued operators in $b$-metric spaces.

A new space called the complex valued metric space which is more general than the well-known metric space has been introduced by Azam et al. [4]. They proved some fixed point results for a pair of mappings for contraction condition satisfying a rational expression. Azam et al. [4] improved the Banach contraction principle in the context of complex valued metric space involving rational inequality which could not be meaningful in cone metric spaces. Several authors studied many common fixed point theorems on complex valued metric spaces (see [5-9]).
The concept of complex valued $b$-metric spaces was introduced in 2013 by Rao et al. [10]. In sequel, Mukheimer [11] proved some common fixed point theorems in complex valued $b$-metric spaces.

In this paper, we continue the study of fixed point theorems in complex valued $b$-metric spaces. The obtained results are generalizations of recent results proved by Dubey et al. [12, 13], Nashine et al. [5, 6], and Rao et al. [10].

\section{Preliminaries}

Let $\mathbb{C}$ be the set of complex numbers and $z_{1}, z_{2} \in \mathbb{C}$. Define a partial order $\lesssim$ on $\mathbb{C}$ as follows:

$z_{1} \preccurlyeq z_{2}$ if and only if $\operatorname{Re}\left(z_{1}\right) \leq \operatorname{Re}\left(z_{2}\right), \operatorname{Im}\left(z_{1}\right) \leq \operatorname{Im}\left(z_{2}\right)$.

Thus $z_{1} \lesssim z_{2}$ if one of the following holds:

(1) $\operatorname{Re}\left(z_{1}\right)=\operatorname{Re}\left(z_{2}\right)$ and $\operatorname{Im}\left(z_{1}\right)=\operatorname{Im}\left(z_{2}\right)$;

(2) $\operatorname{Re}\left(z_{1}\right)<\operatorname{Re}\left(z_{2}\right)$ and $\operatorname{Im}\left(z_{1}\right)=\operatorname{Im}\left(z_{2}\right)$;

(3) $\operatorname{Re}\left(z_{1}\right)=\operatorname{Re}\left(z_{2}\right)$ and $\operatorname{Im}\left(z_{1}\right)<\operatorname{Im}\left(z_{2}\right)$;

(4) $\operatorname{Re}\left(z_{1}\right)<\operatorname{Re}\left(z_{2}\right)$ and $\operatorname{Im}\left(z_{1}\right)<\operatorname{Im}\left(z_{2}\right)$.

We will write $z_{1} \precsim z_{2}$ if $z_{1} \neq z_{2}$ and one of (2), (3), and (4) is satisfied; also we will write $z_{1} \prec z_{2}$ if only (4) is satisfied.

It follows that

(i) $0 \precsim z_{1} \precsim z_{2}$ implies $\left|z_{1}\right|<\left|z_{2}\right|$;

(ii) $z_{1} \precsim z_{2}$ and $z_{2} \prec z_{3}$ imply $z_{1} \prec z_{3}$; 
(iii) $0 \lesssim z_{1} \lesssim z_{2}$ implies $\left|z_{1}\right| \leq\left|z_{2}\right|$;

(iv) if $a, b \in \mathbb{R}, 0 \leq a \leq b$ and $z_{1} \precsim z_{2}$, then $a z_{1} \precsim b z_{2}$ for all $z_{1}, z_{2} \in \mathbb{C}$.

The following definition is recently introduced by Rao et al. [10].

Definition 1. Let $X$ be a nonempty set and let $s \geq 1$ be a given real number. A function $d: X \times X \rightarrow \mathbb{C}$ is called a complex valued $b$-metric on $X$ if for all $x, y, z \in X$ the following conditions are satisfied:

(i) $0 \precsim d(x, y)$ and $d(x, y)=0$ if and only if $x=y$;

(ii) $d(x, y)=d(y, x)$;

(iii) $d(x, y) \precsim s[d(x, z)+d(z, y)]$.

The pair $(X, d)$ is called a complex valued $b$-metric space.

Example 2 (see [10]). If $X=[0,1]$, define the mapping $d$ : $X \times X \rightarrow \mathbb{C}$ by $d(x, y)=|x-y|^{2}+i|x-y|^{2}$, for all $x, y \in X$.

Then $(X, d)$ is complex valued $b$-metric space with $s=2$.

Definition 3 (see [10]). Let $(X, d)$ be a complex valued $b$ metric space.

(i) A point $x \in X$ is called interior point of a set $A \subseteq X$ whenever there exists $0 \prec r \in \mathbb{C}$ such that $B(x, r)=$ $\{y \in X: d(x, y) \prec r\} \subseteq A$.

(ii) A point $x \in X$ is called a limit point of a set $A$ whenever for every $0 \prec r \in \mathbb{C}, B(x, r) \cap(A-\{x\}) \neq \phi$.

(iii) A subset $A \subseteq X$ is called open whenever each element of $A$ is an interior point of $A$.

(iv) A subset $A \subseteq X$ is called closed whenever each element of $A$ belongs to $A$.

(v) A subbasis for a Hausdorff topology $\tau$ on $X$ is a family $F=\{B(x, r): x \in X$ and $0 \prec r\}$.

Definition 4 (see [10]). Let $(X, d)$ be a complex valued $b$ metric space and let $\left\{x_{n}\right\}$ be a sequence in $X$ and $x \in X$.

(i) If for every $c \in \mathbb{C}$, with $0 \prec r$, there is $N \in \mathbb{N}$ such that for all $n>N, d\left(x_{n}, x\right) \prec c$, then $\left\{x_{n}\right\}$ is said to be convergent, $\left\{x_{n}\right\}$ converges to $x$, and $x$ is the limit point of $\left\{x_{n}\right\}$. We denote this by $\lim _{n \rightarrow \infty} x_{n}=x$ or $\left\{x_{n}\right\} \rightarrow x$ as $n \rightarrow \infty$.

(ii) If for every $c \in \mathbb{C}$, with $0 \prec r$, there is $N \in \mathbb{N}$ such that for all $n>N, d\left(x_{n}, x_{n+m}\right) \prec c$, where $m \in \mathbb{N}$, then $\left\{x_{n}\right\}$ is said to be Cauchy sequence.

(iii) If every Cauchy sequence in $X$ is convergent, then $(X, d)$ is said to be a complete complex valued $b$ metric space.

Lemma 5 (see [10]). Let $(X, d)$ be a complex valued b-metric space and let $\left\{x_{n}\right\}$ be a sequence in $X$. Then $\left\{x_{n}\right\}$ converges to $x$ if and only if $\left|d\left(x_{n}, x\right)\right| \rightarrow 0$ as $n \rightarrow \infty$.

Lemma 6 (see [10]). Let $(X, d)$ be a complex valued b-metric space and let $\left\{x_{n}\right\}$ be a sequence in $X$. Then $\left\{x_{n}\right\}$ is a Cauchy sequence if and only if $\left|d\left(x_{n}, x_{n+m}\right)\right| \rightarrow 0$ as $n \rightarrow \infty$, where $m \in \mathbb{N}$.

\section{Main Results}

Theorem 7. Let $(X, d)$ be a complete complex valued $b$-metric space with the coefficient $s \geq 1$ and let $S, T: X \rightarrow X$ be mappings satisfying

$$
\begin{aligned}
& d(S x, T y) \lesssim \alpha d(x, y) \\
& +\frac{\beta d(x, S x) d(y, T y)}{d(x, T y)+d(y, S x)+d(x, y)}
\end{aligned}
$$

for all $x, y \in X$, such that $x \neq y, d(x, T y)+d(y, S x)+d(x, y) \neq$ 0 , where $\alpha, \beta$ are nonnegative reals with $\alpha+s \beta<1$ or $d(S x, T y)=0$ if $d(x, T y)+d(y, S x)+d(x, y)=0$.

Then $S$ and $T$ have a unique common fixed point.

Proof. For any arbitrary point $x_{0} \in X$, define sequence $\left\{x_{n}\right\}$ in $X$ such that

$$
\begin{aligned}
& x_{2 n+1}=S x_{2 n}, \\
& x_{2 n+2}=T x_{2 n+1}
\end{aligned}
$$

for $n=0,1,2,3, \ldots$

Now, we show that the sequence $\left\{x_{n}\right\}$ is Cauchy.

Let $x=x_{2 n}$ and $y=x_{2 n+1}$ in (1); we have

$$
\begin{aligned}
& d\left(x_{2 n+1}, x_{2 n+2}\right) \\
& =d\left(S x_{2 n}, T x_{2 n+1}\right) \precsim \alpha d\left(x_{2 n}, x_{2 n+1}\right) \\
& \quad+\frac{\beta d\left(x_{2 n}, S x_{2 n}\right) d\left(x_{2 n+1}, T x_{2 n+1}\right)}{d\left(x_{2 n}, T x_{2 n+1}\right)+d\left(x_{2 n+1}, S x_{2 n}\right)+d\left(x_{2 n}, x_{2 n+1}\right)} \\
& =\alpha d\left(x_{2 n}, x_{2 n+1}\right) \\
& \quad+\frac{\beta d\left(x_{2 n}, x_{2 n+1}\right) d\left(x_{2 n+1}, x_{2 n+2}\right)}{d\left(x_{2 n}, x_{2 n+2}\right)+d\left(x_{2 n+1}, x_{2 n+1}\right)+d\left(x_{2 n}, x_{2 n+1}\right)}
\end{aligned}
$$

so that

$$
\begin{aligned}
&\left|d\left(x_{2 n+1}, x_{2 n+2}\right)\right| \\
& \leq \alpha\left|d\left(x_{2 n}, x_{2 n+1}\right)\right| \\
&+\frac{\beta\left|d\left(x_{2 n}, x_{2 n+1}\right)\right|\left|d\left(x_{2 n+1}, x_{2 n+2}\right)\right|}{\left|d\left(x_{2 n}, x_{2 n+2}\right)\right|+\left|d\left(x_{2 n}, x_{2 n+1}\right)\right|} .
\end{aligned}
$$

As (owing to triangular inequality)

$$
\left|d\left(x_{2 n+1}, x_{2 n+2}\right)\right| \leq\left|d\left(x_{2 n+1}, x_{2 n}\right)\right|+\left|d\left(x_{2 n}, x_{2 n+2}\right)\right|
$$

therefore

$$
\begin{aligned}
\left|d\left(x_{2 n+1}, x_{2 n+2}\right)\right| \leq & \alpha\left|d\left(x_{2 n}, x_{2 n+1}\right)\right| \\
& +\beta\left|d\left(x_{2 n}, x_{2 n+1}\right)\right| \\
= & (\alpha+\beta)\left|d\left(x_{2 n}, x_{2 n+1}\right)\right| \\
\left|d\left(x_{2 n+1}, x_{2 n+2}\right)\right| \leq & (\alpha+\beta)\left|d\left(x_{2 n}, x_{2 n+1}\right)\right| .
\end{aligned}
$$


Similarly, we obtain

$$
\left|d\left(x_{2 n+2}, x_{2 n+3}\right)\right| \leq(\alpha+\beta)\left|d\left(x_{2 n+1}, x_{2 n+2}\right)\right| .
$$

Since $\alpha+s \beta<1$ and $s \geq 1$, we get $\alpha+\beta<1$. Therefore, with $\delta=\alpha+\beta<1$, and for all $n \geq 0$, and consequently, we have

$$
\begin{aligned}
\left|d\left(x_{2 n+1}, x_{2 n+2}\right)\right| & \leq \delta\left|d\left(x_{2 n}, x_{2 n+1}\right)\right| \\
& \leq \delta^{2}\left|d\left(x_{2 n-1}, x_{2 n}\right)\right| \leq \cdots \\
& \leq \delta^{2 n+1}\left|d\left(x_{0}, x_{1}\right)\right| .
\end{aligned}
$$

That is,

$$
\begin{aligned}
\left|d\left(x_{n+1}, x_{n+2}\right)\right| & \leq \delta\left|d\left(x_{n}, x_{n+1}\right)\right| \leq \delta^{2}\left|d\left(x_{n-1}, x_{n}\right)\right| \\
& \leq \cdots \leq \delta^{n+1}\left|d\left(x_{0}, x_{1}\right)\right| .
\end{aligned}
$$

Thus, for any $m>n, m, n \in \mathbb{N}$, we have

$$
\begin{aligned}
\left|d\left(x_{n}, x_{m}\right)\right| \leq & s\left|d\left(x_{n}, x_{n+1}\right)\right|+s\left|d\left(x_{n+1}, x_{m}\right)\right| \\
\leq & s\left|d\left(x_{n}, x_{n+1}\right)\right|+s^{2}\left|d\left(x_{n+1}, x_{n+2}\right)\right| \\
& +s^{2}\left|d\left(x_{n+2}, x_{m}\right)\right| \\
\leq & s\left|d\left(x_{n}, x_{n+1}\right)\right|+s^{2}\left|d\left(x_{n+1}, x_{n+2}\right)\right| \\
& +s^{3}\left|d\left(x_{n+2}, x_{n+3}\right)\right|+s^{3}\left|d\left(x_{n+3}, x_{m}\right)\right| \\
\leq & \cdots \\
\leq & s\left|d\left(x_{n}, x_{n+1}\right)\right|+s^{2}\left|d\left(x_{n+1}, x_{n+2}\right)\right| \\
& +s^{3}\left|d\left(x_{n+2}, x_{n+3}\right)\right|+\cdots \\
& +s^{m-n-2}\left|d\left(x_{m-3}, x_{m-2}\right)\right| \\
& +s^{m-n-1}\left|d\left(x_{m-2}, x_{m-1}\right)\right| \\
& +s^{m-n}\left|d\left(x_{m-1}, x_{m}\right)\right| .
\end{aligned}
$$

By using (9), we get

$$
\begin{aligned}
\left|d\left(x_{n}, x_{m}\right)\right| \leq & s \delta^{n}\left|d\left(x_{0}, x_{1}\right)\right|+s^{2} \delta^{n+1}\left|d\left(x_{0}, x_{1}\right)\right| \\
& +s^{3} \delta^{n+2}\left|d\left(x_{0}, x_{1}\right)\right|+\cdots \\
& +s^{m-n-2} \delta^{m-3}\left|d\left(x_{0}, x_{1}\right)\right| \\
& +s^{m-n-1} \delta^{m-2}\left|d\left(x_{0}, x_{1}\right)\right| \\
& +s^{m-n} \delta^{m-1}\left|d\left(x_{0}, x_{1}\right)\right| \\
= & \sum_{i=1}^{m-n} s^{i} \delta^{i+n-1}\left|d\left(x_{0}, x_{1}\right)\right|
\end{aligned}
$$

Therefore,

$$
\begin{aligned}
\left|d\left(x_{n}, x_{m}\right)\right| & \leq \sum_{i=1}^{m-n} s^{i+n-1} \delta^{i+n-1}\left|d\left(x_{0}, x_{1}\right)\right| \\
& =\sum_{t=n}^{m-1} s^{t} \delta^{t}\left|d\left(x_{0}, x_{1}\right)\right| \\
& \leq \sum_{t=n}^{\infty}(s \delta)^{t}\left|d\left(x_{0}, x_{1}\right)\right| \\
& =\frac{(s \delta)^{n}}{1-s \delta}\left|d\left(x_{0}, x_{1}\right)\right|
\end{aligned}
$$

and hence

$$
\left|d\left(x_{n}, x_{m}\right)\right| \leq \frac{(s \delta)^{n}}{1-s \delta}\left|d\left(x_{0}, x_{1}\right)\right| \longrightarrow 0
$$

as $m, n \longrightarrow \infty$.

Thus, $\left\{x_{n}\right\}$ is a Cauchy sequence in $X$. Since $X$ is complete, there exists some $u \in X$ such that $x_{n} \rightarrow u$ as $n \rightarrow \infty$. Assume not, then there exists $z \in X$ such that

$$
|d(u, S u)|=|z|>0 .
$$

So by using the triangular inequality and (1), we get

$$
\begin{aligned}
z= & d(u, S u) \lesssim s d\left(u, x_{2 n+2}\right)+s d\left(x_{2 n+2}, S u\right) \\
= & s d\left(u, x_{2 n+2}\right)+s d\left(T x_{2 n+1}, S u\right) \\
\preccurlyeq & s d\left(u, x_{2 n+2}\right)+s \alpha d\left(u, x_{2 n+1}\right) \\
& \quad+\frac{s \beta d(u, S u) d\left(x_{2 n+1}, T x_{2 n+1}\right)}{d\left(u, T x_{2 n+1}\right)+d\left(x_{2 n+1}, S u\right)+d\left(u, x_{2 n+1}\right)} \\
= & s d\left(u, x_{2 n+2}\right)+s \alpha d\left(u, x_{2 n+1}\right) \\
& +\frac{s \beta d(u, S u) d\left(x_{2 n+1}, x_{2 n+2}\right)}{d\left(u, x_{2 n+2}\right)+d\left(x_{2 n+1}, S u\right)+d\left(u, x_{2 n+1}\right)}
\end{aligned}
$$

which implies that

$$
\begin{aligned}
|z|= & |d(u, S u)| \\
\leq & s\left|d\left(u, x_{2 n+2}\right)\right|+s \alpha\left|d\left(u, x_{2 n+1}\right)\right| \\
& +\frac{s \beta|d(u, S u)|\left|d\left(x_{2 n+1}, x_{2 n+2}\right)\right|}{\left|d\left(u, x_{2 n+2}\right)\right|+\left|d\left(x_{2 n+1}, S u\right)\right|+\left|d\left(u, x_{2 n+1}\right)\right|} .
\end{aligned}
$$

Taking the limit of (16) as $n \rightarrow \infty$, we obtain that $|z|=$ $|d(u, S u)| \leq 0$, a contradiction with (14). So $|z|=0$. Hence $S u=u$. Similarly, we obtain $T u=u$.

Now we show that $S$ and $T$ have unique common fixed point of $S$ and $T$. To prove this, assume that $u^{*}$ is another common fixed point of $S$ and $T$. Then

$$
\begin{aligned}
d\left(u, u^{*}\right)= & d\left(S u, T u^{*}\right) \precsim \alpha d\left(u, u^{*}\right) \\
& +\frac{\beta d(u, S u) d\left(u^{*}, T u^{*}\right)}{d\left(u, T u^{*}\right)+d\left(u^{*}, S u\right)+d\left(u, u^{*}\right)}
\end{aligned}
$$


so that

$$
\begin{aligned}
& \left|d\left(u, u^{*}\right)\right| \\
& \quad \leq \alpha\left|d\left(u, u^{*}\right)\right| \\
& \quad+\frac{\beta|d(u, S u)|\left|d\left(u^{*}, T u^{*}\right)\right|}{\left|d\left(u, T u^{*}\right)\right|+\left|d\left(u^{*}, S u\right)\right|+\left|d\left(u, u^{*}\right)\right|} \\
& \quad \lesssim \alpha\left|d\left(u, u^{*}\right)\right|
\end{aligned}
$$

so that $u=u^{*}$ which proves the uniqueness of common fixed point.

Now, we consider the second case: $d(x, T y)+d(y, S x)+$ $d(x, y)=0$. Put $x=x_{2 n}$ and $y=x_{2 n+1}$ in this expression; we get $d\left(x_{2 n}, T x_{2 n+1}\right)+d\left(x_{2 n+1}, S x_{2 n}\right)+d\left(x_{2 n}, x_{2 n+1}\right)=0$ (for any $n)$ which implies $d\left(S x_{2 n}, T x_{2 n+1}\right)=0$ so that $x_{2 n}=S x_{2 n}=$ $x_{2 n+1}=T x_{2 n+1}=x_{2 n+2}$. Thus, we have $x_{2 n+1}=S x_{2 n}=x_{2 n}$, so there exist $K_{1}$ and $l_{1}$ such that $K_{1}=S l_{1}=l_{1}$, where $K_{1}=x_{2 n+1}$ and $l_{1}=x_{2 n}$. Using foregoing arguments, one can also show that there exist $K_{2}$ and $l_{2}$ such that $K_{2}=T l_{2}=l_{2}$, where $K_{2}=$ $x_{2 n+2}$ and $l_{2}=x_{2 n+1}$. As $d\left(l_{1}, T l_{2}\right)+d\left(l_{2}, S l_{1}\right)+d\left(l_{1}, l_{2}\right)=0$ (due to definition) implies $d\left(S l_{1}, T l_{2}\right)=0$, therfore $K_{1}=S l_{1}=$ $T l_{2}=K_{2}$. Thus we obtain that $K_{1}=S l_{1}=S K_{1}$. Similarly, one can also have $K_{2}=T K_{2}$. As $K_{1}=K_{2}$ implies $S K_{1}=T K_{1}=$ $K_{1}$, therefore $K_{1}=K_{2}$ is common fixed point of $S$ and $T$. For uniqueness of common fixed point, assume that $K_{1}^{*}$ in $X$ is another common fixed point of $S$ and $T$. Then we have $S K_{1}^{*}=T K_{1}^{*}=K_{1}^{*}$.

As $d\left(K_{1}, T K_{1}^{*}\right)+d\left(K_{1}^{*}, S K_{1}\right)+d\left(K_{1}, K_{1}^{*}\right)=0$, therefore $d\left(K_{1}, K_{1}^{*}\right)=d\left(S K_{1}, T K_{1}^{*}\right)=0$.

This implies that $K_{1}=K_{1}^{*}$. This completes the proof of the theorem.

Corollary 8 (see [12]). Let $(X, d)$ be a complete complex valued $b$-metric space with the coefficient $s \geq 1$. Let $T: X \rightarrow X$ be a mapping satisfying

$$
\begin{aligned}
& d(T x, T y) \precsim \alpha d(x, y) \\
& \quad+\frac{\beta d(x, T x) d(y, T y)}{d(x, T y)+d(y, T x)+d(x, y)}
\end{aligned}
$$

for all $x, y \in X$ such that $x \neq y, d(x, T y)+d(y, T x)+d(x, y) \neq$ 0 , where $\alpha, \beta$ are nonnegative reals with $\alpha+s \beta<1$ or $d(T x, T y)=0$ if $d(x, T y)+d(y, T x)+d(x, y)=0$. Then $T$ has a unique fixed point in $X$.

Proof. We can prove this result by applying Theorem 7 with $S=T$.

Corollary 9 (see [12]). Let $(X, d)$ be a complete complex valued $b$-metric space with the coefficient $s \geq 1$ and let $T$ : $X \rightarrow X$ be a mapping satisfying (for some fixed $n$ )

$$
\begin{aligned}
& d\left(T^{n} x, T^{n} y\right) \precsim \alpha d(x, y) \\
& \quad+\frac{\beta d\left(x, T^{n} x\right) d\left(y, T^{n} y\right)}{d\left(x, T^{n} y\right)+d\left(y, T^{n} x\right)+d(x, y)}
\end{aligned}
$$

for all $x, y \in X$ such that $x \neq y, d(x, T y)+d(y, T x)+d(x, y) \neq$ 0 , where $\alpha, \beta$ are nonnegative reals with $\alpha+s \beta<1$ or
$d\left(T^{n} x, T^{n} y\right)=0$ if $d\left(x, T^{n} y\right)+d\left(y, T^{n} x\right)+d(x, y)=0$. Then $T$ has a unique fixed point in $X$.

Theorem 10. Let $(X, d)$ be a complete complex valued b-metric space with the coefficient $s \geq 1$ and let $S, T: X \rightarrow X$ be mappings satisfying

$$
\begin{aligned}
& d(S x, T y) \precsim \alpha d(x, y)+\frac{\beta\left[d^{2}(x, T y)+d^{2}(y, S x)\right]}{d(x, T y)+d(y, S x)} \\
& \quad+\gamma[d(x, S x)+d(y, T y)]
\end{aligned}
$$

for all $x, y \in X$ such that $x \neq y$, where $\alpha, \beta$, and $\gamma$ are nonnegative reals with $\alpha+2 s \beta+2 \gamma<1$ or $d(S x, T y)=0$ if $d(x, T y)+d(y, S x)=0$. Then $S$ and $T$ have a unique common fixed point in $X$.

Proof. For any arbitrary point $x_{0} \in X$, define sequence $\left\{x_{n}\right\}$ in $X$ such that

$$
\begin{aligned}
& x_{2 n+1}=S x_{2 n}, \\
& x_{2 n+2}=T x_{2 n+1},
\end{aligned}
$$

$$
\text { for } n=0,1,2, \ldots \text {. }
$$

Now, we show that the sequence $\left\{x_{n}\right\}$ is a Cauchy sequence. Let $x=x_{2 n}$ and $y=x_{2 n+1}$ in (21); we have

$$
\begin{aligned}
d\left(x_{2 n+1}, x_{2 n+2}\right)=d\left(S x_{2 n}, T x_{2 n+1}\right) & \vdots \\
\lesssim & d\left(x_{2 n}, x_{2 n+1}\right) \\
& +\frac{\beta\left[d^{2}\left(x_{2 n}, T x_{2 n+1}\right)+d^{2}\left(x_{2 n+1}, S x_{2 n}\right)\right]}{d\left(x_{2 n}, T x_{2 n+1}\right)+d\left(x_{2 n+1}, S x_{2 n}\right)} \\
& +\gamma\left[d\left(x_{2 n}, S x_{2 n}\right)+d\left(x_{2 n+1}, T x_{2 n+1}\right)\right] \\
= & \alpha d\left(x_{2 n}, x_{2 n+1}\right) \\
& +\frac{\beta\left[d^{2}\left(x_{2 n}, x_{2 n+2}\right)+d^{2}\left(x_{2 n+1}, x_{2 n+1}\right)\right]}{d\left(x_{2 n}, x_{2 n+2}\right)+d\left(x_{2 n+1}, x_{2 n+1}\right)} \\
& +\gamma\left[d\left(x_{2 n}, x_{2 n+1}\right)+d\left(x_{2 n+1}, x_{2 n+2}\right)\right]
\end{aligned}
$$

so that

$$
\begin{aligned}
& \left|d\left(x_{2 n+1}, x_{2 n+2}\right)\right| \leq \alpha\left|d\left(x_{2 n}, x_{2 n+1}\right)\right| \\
& \quad+\frac{\beta\left|d^{2}\left(x_{2 n}, x_{2 n+2}\right)\right|}{\left|d\left(x_{2 n}, x_{2 n+2}\right)\right|} \\
& \quad+\gamma\left[\left|d\left(x_{2 n}, x_{2 n+1}\right)\right|+\left|d\left(x_{2 n+1}, x_{2 n+2}\right)\right|\right] \\
& \left|d\left(x_{2 n+1}, x_{2 n+2}\right)\right| \leq \alpha\left|d\left(x_{2 n}, x_{2 n+1}\right)\right| \\
& \quad+\beta\left|d\left(x_{2 n}, x_{2 n+2}\right)\right| \\
& \quad+\gamma\left[\left|d\left(x_{2 n}, x_{2 n+1}\right)\right|+\left|d\left(x_{2 n+1}, x_{2 n+2}\right)\right|\right] .
\end{aligned}
$$

As

$$
\begin{aligned}
& \left|d\left(x_{2 n}, x_{2 n+2}\right)\right| \\
& \quad \leq s\left[\left|d\left(x_{2 n}, x_{2 n+1}\right)\right|+\left|d\left(x_{2 n+1}, x_{2 n+2}\right)\right|\right],
\end{aligned}
$$


therefore

$$
\begin{aligned}
& \left|d\left(x_{2 n+1}, x_{2 n+2}\right)\right| \\
& \leq \alpha\left|d\left(x_{2 n}, x_{2 n+1}\right)\right| \\
& \quad+s \beta\left[\left|d\left(x_{2 n}, x_{2 n+1}\right)\right|+\left|d\left(x_{2 n+1}, x_{2 n+2}\right)\right|\right] \\
& \quad+\gamma\left[\left|d\left(x_{2 n}, x_{2 n+1}\right)\right|+\left|d\left(x_{2 n+1}, x_{2 n+2}\right)\right|\right] \\
& \left|d\left(x_{2 n+1}, x_{2 n+2}\right)\right| \leq\left(\frac{\alpha+s \beta+\gamma}{1-s \beta-\gamma}\right)\left|d\left(x_{2 n}, x_{2 n+1}\right)\right| .
\end{aligned}
$$

Similarly, we obtain

$$
\left|d\left(x_{2 n+2}, x_{2 n+3}\right)\right| \leq\left(\frac{\alpha+s \beta+\gamma}{1-s \beta-\gamma}\right)\left|d\left(x_{2 n+1}, x_{2 n+2}\right)\right| \text {. }
$$

Since $\alpha+2 s \beta+2 \gamma<1$ and $s \geq 1$ we get $\alpha+2 \beta+2 \gamma<1$.

Therefore, with $\delta=(\alpha+\beta+\gamma) /(1-\beta-\gamma)<1$, and for all $n \geq 0$, and consequently, we have

$$
\begin{aligned}
\left|d\left(x_{2 n+1}, x_{2 n+2}\right)\right| & \leq \delta\left|d\left(x_{2 n}, x_{2 n+1}\right)\right| \\
& \leq \delta^{2}\left|d\left(x_{2 n-1}, x_{2 n}\right)\right| \leq \cdots \\
& \leq \delta^{2 n+1}\left|d\left(x_{0}, x_{1}\right)\right| .
\end{aligned}
$$

That is,

$$
\begin{aligned}
\left|d\left(x_{n+1}, x_{n+2}\right)\right| & \leq \delta\left|d\left(x_{n}, x_{n+1}\right)\right| \leq \delta^{2}\left|d\left(x_{n-1}, x_{n}\right)\right| \\
& \leq \cdots \leq \delta^{n+1}\left|d\left(x_{0}, x_{1}\right)\right| .
\end{aligned}
$$

Thus, for any $m>n, m, n \in \mathbb{N}$, we get

$$
\begin{aligned}
\left|d\left(x_{n}, x_{m}\right)\right| \leq & s\left|d\left(x_{n}, x_{n+1}\right)\right|+s\left|d\left(x_{n+1}, x_{m}\right)\right| \\
\leq & s\left|d\left(x_{n}, x_{n+1}\right)\right|+s^{2}\left|d\left(x_{n+1}, x_{n+2}\right)\right| \\
& +s^{2}\left|d\left(x_{n+2}, x_{m}\right)\right| \\
\leq & s\left|d\left(x_{n}, x_{n+1}\right)\right|+s^{2}\left|d\left(x_{n+1}, x_{n+2}\right)\right| \\
& +s^{3}\left|d\left(x_{n+2}, x_{n+3}\right)\right|+s^{3}\left|d\left(x_{n+3}, x_{m}\right)\right| \\
\leq & \cdots \\
\leq & s\left|d\left(x_{n}, x_{n+1}\right)\right|+s^{2}\left|d\left(x_{n+1}, x_{n+2}\right)\right| \\
& +s^{3}\left|d\left(x_{n+2}, x_{n+3}\right)\right|+\cdots \\
& +s^{m-n-2}\left|d\left(x_{m-3}, x_{m-2}\right)\right| \\
& +s^{m-n-1}\left|d\left(x_{m-2}, x_{m-1}\right)\right| \\
& +s^{m-n}\left|d\left(x_{m-1}, x_{m}\right)\right| .
\end{aligned}
$$

By using (29) we get

$$
\begin{aligned}
\left|d\left(x_{n}, x_{m}\right)\right| \leq & s \delta^{n}\left|d\left(x_{0}, x_{1}\right)\right|+s^{2} \delta^{n+1}\left|d\left(x_{0}, x_{1}\right)\right| \\
& +s^{3} \delta^{n+2}\left|d\left(x_{0}, x_{1}\right)\right|+\cdots \\
& +s^{m-n-2} \delta^{m-3}\left|d\left(x_{0}, x_{1}\right)\right| \\
& +s^{m-n-1} \delta^{m-2}\left|d\left(x_{0}, x_{1}\right)\right| \\
& +s^{m-n} \delta^{m-1}\left|d\left(x_{0}, x_{1}\right)\right| \\
= & \sum_{i=1}^{m-n} s^{i} \delta^{i+n-1}\left|d\left(x_{0}, x_{1}\right)\right| .
\end{aligned}
$$

Therefore

$$
\begin{aligned}
\left|d\left(x_{n}, x_{m}\right)\right| & \leq \sum_{i=1}^{m-n} s^{i+n-1} \delta^{i+n-1}\left|d\left(x_{0}, x_{1}\right)\right| \\
& =\sum_{t=n}^{m-1} s^{t} \delta^{t}\left|d\left(x_{0}, x_{1}\right)\right| \\
& \leq \sum_{t=n}^{\infty}(s \delta)^{t}\left|d\left(x_{0}, x_{1}\right)\right| \\
& =\frac{(s \delta)^{n}}{1-s \delta}\left|d\left(x_{0}, x_{1}\right)\right|
\end{aligned}
$$

and hence

$$
\left|d\left(x_{n}, x_{m}\right)\right| \leq \frac{(s \delta)^{n}}{1-s \delta}\left|d\left(x_{0}, x_{1}\right)\right| \longrightarrow 0
$$

as $m, n \longrightarrow \infty$.

Thus, $\left\{x_{n}\right\}$ is a Cauchy sequence in $X$. Since $X$ is complete, there exists some $u \in X$ such that $x_{n} \rightarrow u$ as $n \rightarrow \infty$. Assume not, then there exists $z \in X$ such that

$$
|d(u, S u)|=|z|>0 .
$$

So by using the triangular inequality and (21), we get

$$
\begin{aligned}
z= & d(u, S u) \lesssim s d\left(u, x_{2 n+2}\right)+s d\left(x_{2 n+2}, S u\right) \\
= & s d\left(u, x_{2 n+2}\right)+s d\left(S u, T x_{2 n+1}\right) \\
\lesssim & s d\left(u, x_{2 n+2}\right)+s \alpha d\left(u, x_{2 n+1}\right) \\
& +\frac{s \beta\left[d^{2}\left(u, T x_{2 n+1}\right)+d^{2}\left(x_{2 n+1}, S u\right)\right]}{d\left(u, T x_{2 n+1}\right)+d\left(x_{2 n+1}, S u\right)} \\
& +s \gamma\left[d(u, S u)+d\left(x_{2 n+1}, T x_{2 n+1}\right)\right] \\
= & s d\left(u, x_{2 n+2}\right)+s \alpha d\left(u, x_{2 n+1}\right) \\
& +\frac{s \beta\left[d^{2}\left(u, x_{2 n+2}\right)+d^{2}\left(x_{2 n+1}, S u\right)\right]}{d\left(u, x_{2 n+2}\right)+d\left(x_{2 n+1}, S u\right)} \\
& +s \gamma\left[z+d\left(x_{2 n+1}, x_{2 n+2}\right)\right]
\end{aligned}
$$


which implies that

$$
\begin{aligned}
|z|= & |d(u, S u)| \\
\leq & s\left|d\left(u, x_{2 n+2}\right)\right|+s \alpha\left|d\left(u, x_{2 n+1}\right)\right| \\
& +\frac{s \beta\left[\left|d^{2}\left(u, x_{2 n+2}\right)\right|+\left|d^{2}\left(x_{2 n+1}, S u\right)\right|\right]}{\left|d\left(u, x_{2 n+2}\right)\right|+\left|d\left(x_{2 n+1}, S u\right)\right|} \\
& +s \gamma\left[|z|+\left|d\left(x_{2 n+1}, x_{2 n+2}\right)\right|\right] .
\end{aligned}
$$

Taking the limit of (36) as $n \rightarrow \infty$, we obtain that $|z|=$ $|d(u, S u)| \leq 0$, a contradiction with (34). So $|z|=0$; hence $S u=u$.

Similarly, we obtain that $T u=u$. Now, we show that $S$ and $T$ have a unique common fixed point of $S$ and $T$. To show this, assume that $u^{*}$ is another common fixed point of $S$ and $T$. Then

$$
\begin{aligned}
d\left(u, u^{*}\right)= & d\left(S u, T u^{*}\right) \\
\lesssim & \alpha d\left(u, u^{*}\right) \\
& +\frac{\beta\left[d^{2}\left(u, T u^{*}\right)+d^{2}\left(u^{*}, S u\right)\right]}{d\left(u, T u^{*}\right)+d\left(u^{*}, S u\right)} \\
& +\gamma\left[d(u, S u)+d\left(u^{*}, T u^{*}\right)\right],
\end{aligned}
$$

$\prec d\left(u, u^{*}\right)$, a contradiction. So $u=u^{*}$, which proves the uniqueness of common fixed point in $X$. For the second case $d(S x, T y)=0$ if $d(x, T y)+d(y, S x)=0$, the proof of unique common fixed point can be completed in the line of Theorem 7. This completes the proof of the theorem.

Corollary 11. Let $(X, d)$ be a complete complex valued $b$ metric space with the coefficient $s \geq 1$ and let $T: X \rightarrow X$ be a mapping satisfying

$$
\begin{aligned}
d(T x, T y) \precsim & \alpha d(x, y)+\frac{\beta\left[d^{2}(x, T y)+d^{2}(y, T x)\right]}{d(x, T y)+d(y, T x)} \\
& +\gamma[d(x, T x)+d(y, T y)]
\end{aligned}
$$

for all $x, y \in X$ such that $x \neq y$, where $\alpha, \beta$, and $\gamma$ are nonnegative reals with $\alpha+2 s \beta+2 \gamma<1$ or $d(T x, T y)=0$ if $d(x, T y)+d(y, T x)=0$. Then $T$ has a unique fixed point.

Proof. We can prove this result by applying Theorem 10 with $S=T$.

Corollary 12. Let $(X, d)$ be a complete complex valued $b$ metric space with the coefficient $s \geq 1$ and let $T: X \rightarrow X$ be a mapping satisfying (for some fixed $n$ )

$$
\begin{aligned}
d\left(T^{n} x, T^{n} y\right) \lesssim \alpha d(x, y) \\
+\frac{\beta\left[d^{2}\left(x, T^{n} y\right)+d^{2}\left(y, T^{n} x\right)\right]}{d\left(x, T^{n} y\right)+d\left(y, T^{n} x\right)} \\
+\gamma\left[d\left(x, T^{n} x\right)+d\left(y, T^{n} y\right)\right]
\end{aligned}
$$

for all $x, y \in X$, such that $x \neq y$, where $\alpha, \beta$, and $\gamma$ are nonnegative reals with $\alpha+2 s \beta+2 \gamma<1$ or $d\left(T^{n} x, T^{n} y\right)=0$ if $d\left(x, T^{n} y\right)+d\left(y, T^{n} x\right)=0$. Then $T$ has a unique fixed point in $X$.

Proof. From Corollary 11, we obtain that $u \in X$ such that $T^{n} u=u$. The uniqueness follows from

$$
\begin{aligned}
d(T u, u)= & d\left(T T^{n} u, T^{n} u\right)=d\left(T^{n} T u, T^{n} u\right) \\
\lesssim & \alpha d(T u, u) \\
& +\frac{\beta\left[d^{2}\left(T u, T^{n} u\right)+d^{2}\left(u, T^{n} T u\right)\right]}{d\left(T u, T^{n} u\right)+d\left(u, T^{n} T u\right)} \\
& +\gamma\left[d\left(T u, T^{n} T u\right)+d\left(u, T^{n} u\right)\right] \\
= & (\alpha+2 \beta) d(T u, u) .
\end{aligned}
$$

By Taking modulus of $(40)$ and since $(\alpha+2 \beta)<1$, we obtain $|d(T u, u)| \leq(\alpha+2 \beta)|d(T u, u)|<|d(T u, u)|$, a contradiction. So $T u=u$. Hence $T u=T^{n} u=u$. Therefore, the fixed point of $T$ is unique. This completes the proof.

\section{Competing Interests}

The author declares that they have no competing interests.

\section{References}

[1] S. Banach, "Sur les operations dans les ensembles abstraits et leur application aux equations integrals," Fundamenta Mathematicae, vol. 3, pp. 133-181, 1922.

[2] I. A. Bakhtin, "The contraction principle in quasi metric spaces," Journal of Functional Analysis, vol. 30, pp. 26-37, 1989.

[3] S. Czerwik, "Nonlinear set-valued contraction mappings in b-metric spaces," Atti del Seminario Matematico e Fisico dell'Università di Modena, vol. 46, no. 2, pp. 263-276, 1998.

[4] A. Azam, B. Fisher, and M. Khan, "Common fixed point theorems in complex valued metric spaces," Numerical Functional Analysis and Optimization, vol. 32, no. 3, pp. 243-253, 2011.

[5] H. K. Nashine, M. Imdad, and M. Hasan, "Common fixed point theorems under rational contractions in complex valued metric spaces," Journal of Nonlinear Science and Its Applications, vol. 7, no. 1, pp. 42-50, 2014.

[6] H. K. Nashine, "Common fixed point for a pair of maps in complex valued metric spaces," Nonlinear Functional Analysis and Applications, vol. 18, no. 2, pp. 279-289, 2013.

[7] F. Rouzkard and M. Imdad, "Some common fixed point theorems on complex valued metric spaces," Computers \& Mathematics with Applications, vol. 64, no. 6, pp. 1866-1874, 2012.

[8] W. Sintunavarat and P. Kumam, "Generalized common fixed point theorems in complex valued metric spaces and applications," Journal of Inequalities and Applications, vol. 2012, article 84, 11 pages, 2012.

[9] W. Sintunavarat, Y. J. Cho, and P. Kumam, "Urysohn integral equations approach by common fixed points in complex-valued metric spaces," Advances in Difference Equations, vol. 2013, article 49, 2013. 
[10] K. P. R. Rao, P. R. Swamy, and J. R. Prasad, "A common fixed point theorem in complex valued b-metric spaces," Bulletin of Mathematics and Statistics Research, vol. 1, no. 1, pp. 1-8, 2013.

[11] A. A. Mukheimer, "Some common fixed point theorems in complex valued $b$-metric spaces," The Scientific World Journal, vol. 2014, Article ID 587825, 6 pages, 2014.

[12] A. K. Dubey, R. Shukla, and R. P. Dubey, "Some fixed point theorems in complex valued b-metric spaces," Journal of Complex Systems, vol. 2015, Article ID 832467, 7 pages, 2015.

[13] A. K. Dubey, "Common fixed point results for contractive mappings in Complex valued b-Metric spaces," Nonlinear Functional Analysis and Applications, vol. 20, no. 2, pp. 257-268, 2015. 


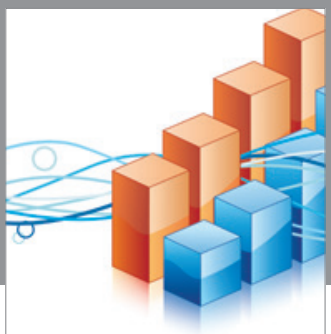

Advances in

Operations Research

vatem alat4

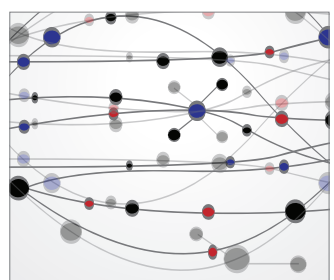

\section{The Scientific} World Journal
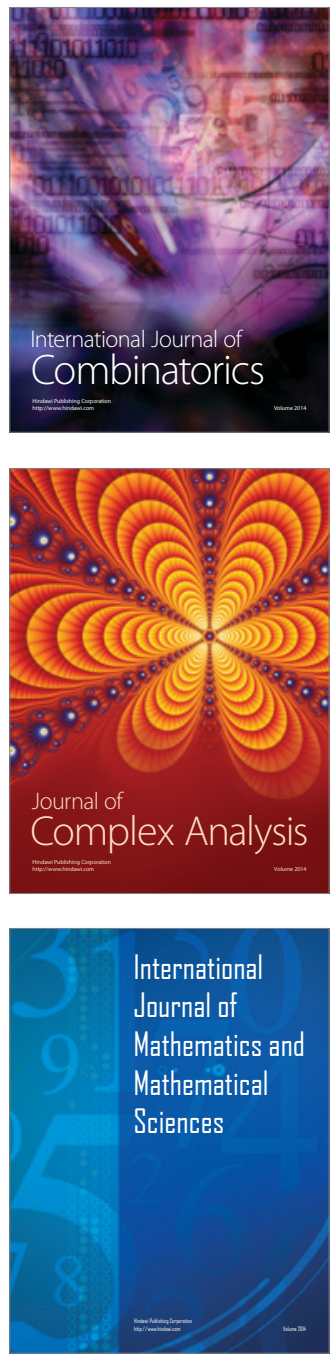
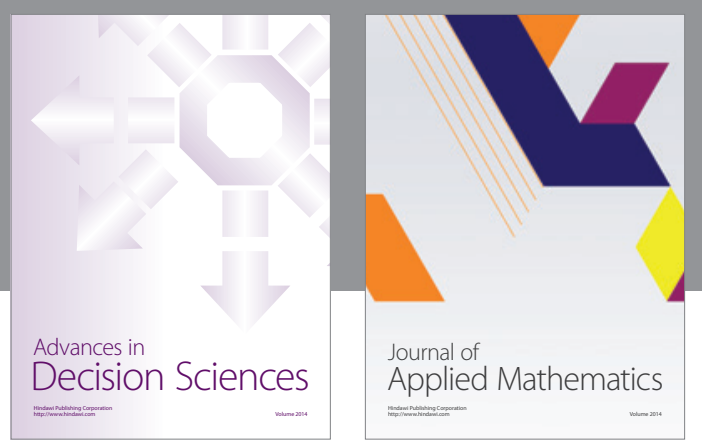

Algebra

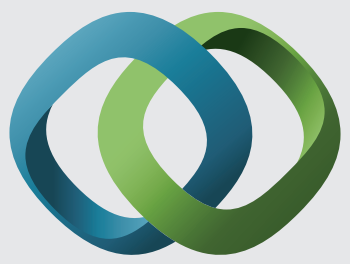

\section{Hindawi}

Submit your manuscripts at

http://www.hindawi.com
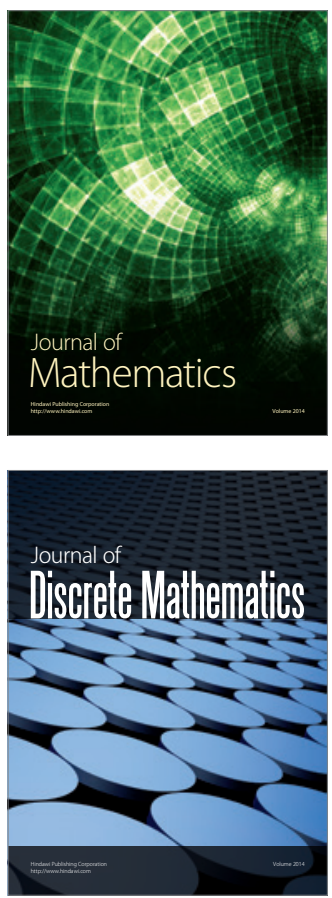

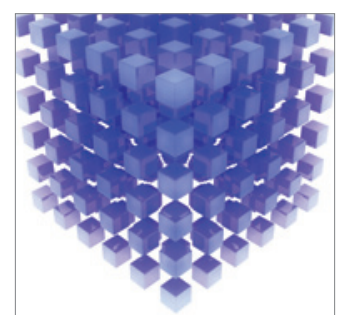

Mathematical Problems in Engineering
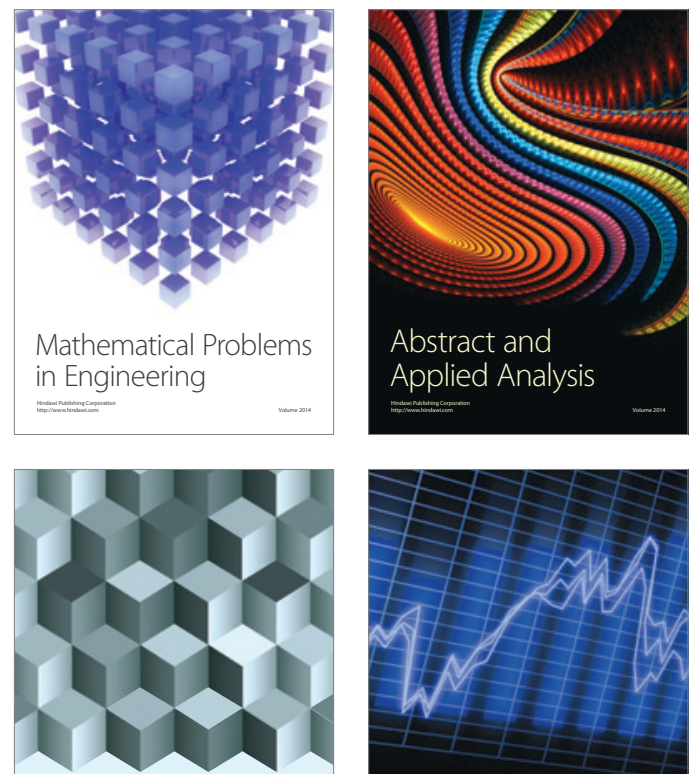

Journal of

Function Spaces

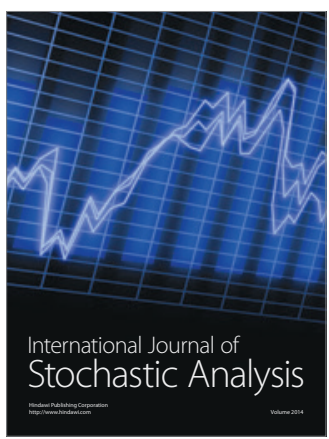

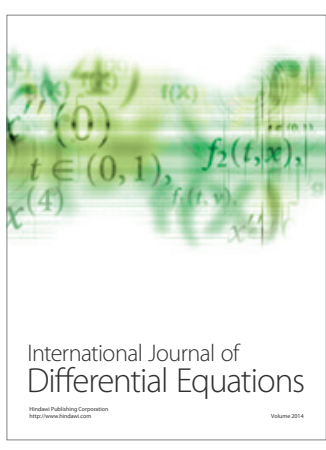
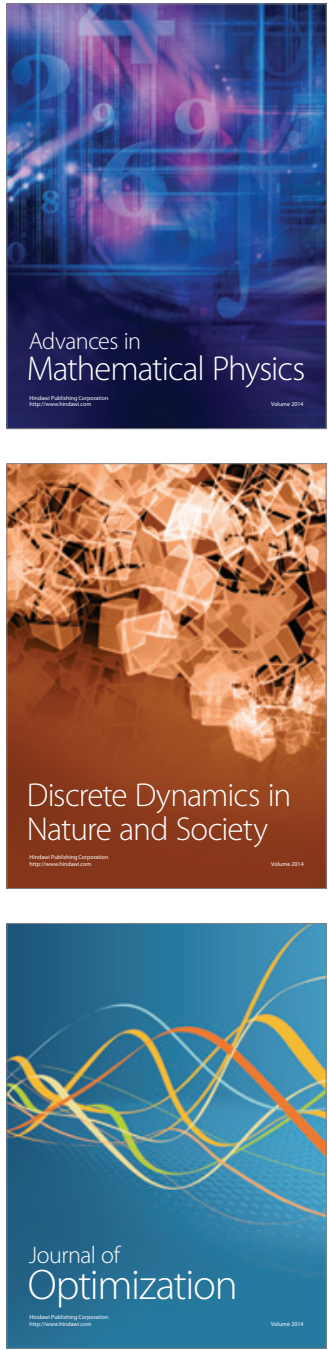